EPJ Web of Conferences 110,01065 (2016)

DOI: $10.1051 /$ epjconf/201611001065

(C) Owned by the authors, published by EDP Sciences, 2016

\title{
THE CALCULATED RATIO OF THE GAS FLOW IN A COUNTERCURRENT CYCLONE DUST CONCENTRATOR
}

\author{
Michail Vasilevsky ${ }^{1}$, Aleksandr Razva ${ }^{1, a}$, Alissa Pleschko ${ }^{1}$, and Ivan Kadurkin ${ }^{1}$ \\ ${ }^{1}$ National research Tomsk polytechnic university, 634050 Tomsk, Russia
}

\begin{abstract}
There are numerous studies of the structure of swirling flow in a variety of devices in which the peculiarities of the parameters associated with the twist flow. The values of the local parameters of the twist of the axial direction are experimentally and connect them with a constructive twist parameter, which is built from the idealized repose of the gas flow in vortex distribution and speed at the exit of the swirl. For counter flow chamber is the equation for the input pulse in the radial direction and the twist parameter is provided in the radial direction. It allows us to estimate the maximum radius of the circumferential velocity not only near the outlet, but also near the end surface of the chamber. On a cylindrical surface with a radius of outlet cyclone tangential turbulent friction in the radial direction depends on the product of a circle and radial speeds. Compiled equation changes the flow of angular momentum in the axial zone, depending on the force of friction tangential flow on the surface with the radius of the outlet pipe of the cyclone. This equation allowed assessing the circulation of gas in the axial zone.
\end{abstract}

\section{Introduction}

Swirling flow of gases are an integral part of many complex flows and are widely distributed in nature and technology. The working process of a number of technical devices (cyclone vortex combustion, power technology, drying, smelting chambers, burners, nebulizers, etc.) is largely determined by the laws of fluid dynamics and heat and mass transfer rotating flows. Tightening the flow in the chamber creates a very uneven radial speed and pressure fields, which promotes intensive mixing environment and phase separation. Elucidation of the laws of motion and flow in vortex cyclone unit devoted thousands of research, but the existing contradictions in the experimental evaluations and interpretations of the processes do not allow sufficient detail to get ideas about the mechanics of traffic flows in co-current and counter-cyclone unit. Typically, the length of these devices does not exceed 5 calibers $\left(\mathrm{LK}_{\mathrm{K}} \mathrm{DK}<5\right)$. We understand by the vortex chambers chamber side gas injection, the length of each of which is equal to the length of the chamber; a cyclone chamber will understand the camera with the end or side entry, the length of which is less than the length of the chamber

\section{Theoretical bases}

There are numerous studies of the structure of torqued flow in a variety of devices in which the peculiarities of the parameters associated with the twist stream [1,3]. The twist given local parameters which define the state of flow in a particular section. The values of the local parameters are experimental twist and tie them with a constructive twist parameter, which is built from the idealized repose of the gas flow in vortex distribution and velocity at the exit of the swirl. For example, to this parameter vane swirl determined trailing edge tangent angle in relation to the axial direction to a tangential or snail input parameter is determined by multiplying the cross-sectional area ratio to the area of the input chamber and the ratio of the mean radius of the channel input to the radius of the cell.

\subsection{Local settings}

Frequently used local parameters twist in the axial direction in the form of $\Phi z=M z / K z R \kappa, \Phi z *=M z / K z * R \kappa$,

$$
M_{z}=2 \pi \int_{0}^{\mathrm{R} \kappa} \rho u w r^{2} d r K_{z}=2 \pi \int_{0}^{\mathrm{RK}}\left(p+\rho w^{2}\right) r d r K_{z}^{*}=2 \pi \int_{0}^{\mathrm{R} \kappa} \rho w^{2} r d r
$$

\footnotetext{
${ }^{\text {a }}$ Corresponding author: razva@mail.ru
} 


\section{EPJ Web of Conferences}

where $\mathrm{r}, \mathrm{R} \kappa$ - radii and the current camera, $\mathrm{p}, \rho$ - excessive static pressure and density of the gas, $\mathrm{v}, \mathrm{w}-$ circumferential and axial velocity.

In the cyclone, vortical cell gas swirl in the periphery and in the central part goes through a channel with a smaller radius. Transfer torqued flow occurs in the direction of the axis in the radial direction, and determines the magnitude of the flow must be a flow of angular momentum in the radial direction and amount of movement in this direction across the surface with the radius of the exhaust outlet. Therefore, the twist parameter should be the expression

$$
\Phi_{\mathrm{r}}=\frac{M_{\mathrm{r}}}{K_{\mathrm{r}} R_{1}}, \Phi_{\mathrm{r}}^{*}=\frac{M_{\mathrm{r}}}{K_{\mathrm{r}}^{*} R_{1}}, M_{r}=2 \pi R_{1} \int_{0}^{\mathrm{Lt}} \rho v u R_{\mathrm{l}} d z, K_{\mathrm{r}}=2 \pi R_{1} \int_{0}^{\mathrm{Lt}}\left(p+\rho u^{2}\right) d z, K_{\mathrm{r}}^{*}=2 \pi R_{1} \int_{0}^{\mathrm{Lt}} \rho u^{2} d z,
$$

where $R_{1}$ - radius exhaust pipe or holes $\mathrm{u}$ - radial speed $\mathrm{z}$ - axled coordinate, $\mathrm{L}_{t}$ - reach camera from the end caps to the bottom section of the gas outlet.

If you put that on the surface with a radius $R_{1}$ velocities $u$, and $v$ are constant,

$$
\Phi_{\mathrm{r}}^{*}=\frac{M_{\mathrm{r}}}{K_{\mathrm{r}}^{*} R_{1}}=\frac{v_{1}}{u_{1}}=\operatorname{ctg} \alpha_{1}, S_{\mathrm{v}}=\mathrm{v}_{1} / w_{\mathrm{cp}}[2]
$$

wherein $v_{1}$ - circumferential speed of the gas at the radius $R_{1}, w_{\mathrm{cp}}$ - mean-feed gas velocity in the exhaust passage. When the potential distribution along the radius $v, v_{1}=v_{\mathrm{K}} R_{\mathrm{K}} / R_{1}$ и $S_{\mathrm{v}}=1 / m_{\mathrm{a}}, m_{a}=f_{\mathrm{Bx}} / \pi R_{1} R_{\mathrm{K}}=\bar{f}_{\mathrm{BX}} / \bar{R}_{1}-$ design parameter twist [4].

There $f_{\mathrm{Bx}}-$ summary entrance area,

$$
\bar{f}_{\mathrm{Bx}}=f_{\mathrm{Bx}} / \pi R_{\mathrm{K}}^{2}, u_{1}=\frac{w_{\mathrm{cp}} \pi R_{1}^{2}}{2 \pi R_{\mathrm{l}} L_{\mathrm{\kappa}}}=w_{\mathrm{cp}} \frac{R_{\mathrm{l}}}{L_{\mathrm{\kappa}}}, \Phi_{\mathrm{r}}^{*}=\frac{v_{1}}{u_{1}}=\operatorname{ctg} \alpha_{1}=S_{\mathrm{v}} \frac{L_{\mathrm{K}}}{R_{1}} .
$$

The last parameter enables the relative length of the chamber and is more versatile than $S_{\mathrm{v}}=1 / m_{\mathrm{a}}$. Comparing the expressions obtained, we find

$$
\mathrm{m}_{a}=\operatorname{tg} \alpha_{1} \frac{\mathrm{L}_{\kappa}}{\mathrm{R}_{1}}
$$

\subsection{Characteristics of streams}

In [5] shows the results of a study on the impact of the length of the chamber pressure loss of circulation and distribution. Moreover, significant changes along the chamber is not observed, while the total circumferential velocity in the chamber and the pressure on the walls decreases. This indicates that the cell is averaged over the length of the circumferential velocity distribution in the radial direction by the radialno-axial and turbulent transfer of momentum and angular movement. The pressure on the wall relative to the pressure input is determined not only by constructional features vortex, but the relative dimensions of the outlet and the relative length of the chamber, which accounts for the effect ejection indirectly effects in the central area, which extends up to the input splines [6]. Effect of generating turbulence in the forced vortex spreads all over, but the $R_{1} / R_{\mathrm{K}}<0,2$ braking rotation freevortex flow slightly and the circumferential velocity distribution of a free vortex close to potential [2].

The main characteristic of the flow is magnitude $v_{\mathrm{m}}$, circumferential velocity, the radius $r_{\mathrm{m}}$, where the circumferential velocity is equal $v_{\mathrm{m}}$, and the radius of the reverse current $r_{0}$, where the axial velocity is zero, and zero overpressure $r_{\mathrm{p} 0}$ [7]. The specific design parameter ranges of these quantities uniquely ma dependent $m_{\mathrm{a}}$ [2]. For example, when $0,2<m_{\mathrm{a}}<2,2$ most satisfactory agreement with the experiment gives the ratio $r_{\mathrm{m}} / R_{1}=0,35 / \sqrt{m_{a}}$ obtained in [8]. When most commonly encountered ratio $L_{\mathrm{K}} / R_{\mathrm{K}}$ $=2, m_{\mathrm{a}}=2 \operatorname{tg} \alpha_{1}$ in the range $0,1<\operatorname{tg} \alpha 1<1,1 r_{\mathrm{m}} / R_{1}=0,175 / \sqrt{\operatorname{tg} \alpha_{1}}$. Near the outlet $\operatorname{tg} \alpha 1 \approx 0,1$ and $r_{\mathrm{m}} / R_{1}=0,175 / \sqrt{0,1}=0,55$.

In the end region of the walls and stands out of the jet, and has a maximum radial velocity, which is comparable in magnitude to the peripheral speed, which is comparable in magnitude to the peripheral speed [5]. In the axial region at the bottom is set $\operatorname{tg} \alpha_{\mathrm{cr}}=u /\left.v\right|_{z \rightarrow 0}, \operatorname{tg} \alpha_{\mathrm{cr}} \approx 1$ и $r_{\mathrm{m}} / R_{1}=0,175 / \sqrt{1}=0,175$. Thus, in the axial region at the bottom of the chamber value $r_{\mathrm{m}} / R_{1}$ is about 3 times less than in the outlet chamber. Significant reduction of the radius of the maximum peripheral speed and the radius of zero excess pressure at the bottom of the camera can be seen on the location of the air chamber in the centrifugal nozzle [9]. The structure of the flow in the bottom region near the axis of the vortex chamber is discussed in [10].

Area quasi potential flow, called the nucleus, is in the range $r_{\mathrm{m}}<r<r_{\mathrm{g}}$, where $r$ s - radius transition from the motion of the gas jet in the curved wall of the potential. This region is formed of the branches of most of the current gas jet and output at the field of the current of gas in the blast area. The central region is in the range $0<r<r_{\mathrm{m}}$. In contrast to the free jet in the axial currents and interaction quasipotential flow dripping gas outlet to the channel where the tangential velocity becomes the maximum value, there 
is the most intensive mixing due to counter radial transport of angular momentum, and the formation of the axial velocity gradients.

\section{Relations for countercurrent cyclone concentrator}

The cyclone dust hubs recessed central pipe inside the chamber, in which the purified gas is supplied by one or several connections (Figure 1). It adopted distributed around the circumference of the gas inlet in order to meet the conditions with respect to the axis of symmetry of the flow [11], although the most commonly used or snail poluulitochny gas inlet [12]. In the area of twist between the input and output slots pipe flow from the radial direction becomes downward axial movement by rotating the reaction gas moles $R_{\mathrm{uw}}$, creating centrifugal forces. This reaction should be equal to the gas flow in the axial direction. In addition, there is the pressure force of the outer surface of the exhaust outlet to the stream. Formed as the radial momentum $R_{\mathrm{uw}}$ of the axial flow coming out of the ring with a radius $r_{0}$ and $R_{1}$. The equation of the momentum flux in the radial direction can be

$$
l_{\mathrm{Bx}} 2 \pi R_{\mathrm{\kappa}}\left(u_{\mathrm{K}}^{2} \rho+P_{\mathrm{\kappa}}\right)=2 \pi \rho \int_{0}^{l_{\mathrm{BX}}} \int_{\mathrm{R}_{1}}^{\mathrm{R}_{\mathrm{K}}} \frac{v^{2}}{r} r d r d z+R_{\mathrm{uw}}+P_{1} l_{\mathrm{Bx}} 2 \pi R_{1}+R_{\mathrm{wu}}
$$

where $-R_{\mathrm{uw}}=2 \pi \rho \int_{r_{0}}^{\mathrm{R}} w^{2} r d r, R_{\mathrm{wu}}=2 \pi \rho \int_{\mathrm{R}_{1}}^{\mathrm{r}_{0}} w^{2} r d r$, Here $r_{0}$ - radius of the surface on which the axial speed is zero; $u_{\mathrm{\kappa}}=\frac{Q}{2 \pi R_{\mathrm{\kappa}} l_{\mathrm{Bx}}}$. The position is determined by the radius $r_{0}$ of twist flow parameter and depends on the total area of the entrance relative the connecting gas pipes.
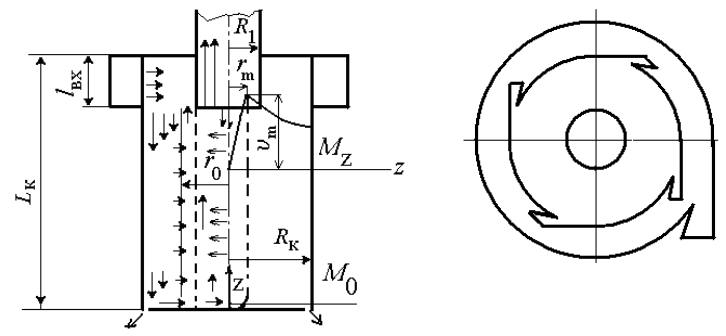

Figure 1. The cyclone concentrator.

Spun jet expands inside. Generating turbulence in contrast to the rectilinear flow in a pipe is carried out not only in the nearwall zone, but also in the whole flow region [13]. Area traffic is quasi-solid zone ejected flow driven by a vortex formed in the machine. The configuration of the axial zone of quasi-solid movement is in the boundary layer of the end tapered. In this zone, the angular velocity of rotation increases with proximity to the bottom. In the exhaust pipe stops the action of the radial motion of the axis and the flow expanded, initiating the return movement near the axis.

In the central region there is a zone quasisolid gas rotation in which the values of the speed components are comparable and the turbulence intensity reaches $40 \%$ [13]. This zone is formed near the end of the jet stream which is formed by the rotation of flow in the boundary layer of the end-face boundary of a radial direction in axial. This rotation is carried out by rotating the reaction of moles of gas, under the influence of centrifugal forces, which are in the axial region at the bottom of the larger values than gas outlet pipe. However, this jet stream localized radial movement of gas toward the axis of the core flow. The equation of change of angular momentum of gas in the central region. The angular momentum with increasing $z$ is increased by the addition of mass (Figure 1) In section 0 axial velocity is determined by the averaged radial velocity in the boundary layer and the mass flow in the boundary layer. In the section $z$ mass flow rate is determined by the amount of gas flowing in section 0 and join the amount of gas between sections 0 and $\mathrm{z}$, as well as the amount of recycled gas in the axial region $Q_{\mathrm{u}}$ equations of motion of the gas for the district components in integral form can be written as in the region $0<r<R_{1}$.

$$
\frac{d M}{d z}=2 \pi R_{1}^{2} \tau_{\mathrm{r} \varphi}, M=2 \pi \rho \int_{0}^{\mathrm{R}_{1}} \mathrm{v} w r^{2} d r=2 \pi \rho \int_{0}^{\mathrm{r}_{\mathrm{m}}} v w r^{2} d r+\int_{\mathrm{r}_{\mathrm{m}}}^{\mathrm{R}_{1}} v w r^{2} d r
$$

$\tau_{\mathrm{r} \varphi}-$ turbulent frictional stress in the circumferential direction of transport of moles of gas in the radial direction [14]. The magnitude of the axial velocity (2) in the area of $\left[r_{\mathrm{m}}, R_{1}\right]$ order more than in the $\left[0, r_{\mathrm{m}}\right]$, and in the axial region are negative currents of gas towards the end [2]. In [15] it is shown that the magnitude of the potential for flat $\tau_{\mathrm{r} \varphi}$ torqued flow can be represented as

$$
\tau_{\mathrm{r} \varphi}=\rho w \mathbf{v}
$$




\section{EPJ Web of Conferences}

We assume that a cylindrical surface with radius $R_{l}$ shear stress (3) is written as $\tau_{\mathrm{r} \varphi 1}=\rho u_{1} v_{1}$.

In these equations, the main variable is the radial component of the velocity, which is less than the order of the district in the bulk chamber. However, turbulent transfer rate $u_{*}=\sqrt{\tau_{\mathrm{r} \varphi} / \rho}$, defined by the relation (3) is much greater than the radial velocity and is therefore a determining factor. The magnitude of the axial angular momentum at a distance $z$ denote $M_{\mathrm{z}}$

We assume that the radial velocity in the cyclone chamber is distributed on the surface of a radius $R_{1}$ evenly height: $u_{1}=\frac{Q-Q_{\mathrm{T}}}{2 \pi R_{1}\left(L_{\mathrm{K}}-l_{\mathrm{BX}}\right)}$ The actual maximum speed in the central region has a stepped form (Figure 1). Mean-feed speed in the gap between the surfaces of the cylinder radii $R_{l}$ and $r_{m}$ to each section is

$$
W_{\mathrm{zQ}}=\frac{2 \pi \mathrm{R}_{1} u_{1} z+Q_{\mathrm{T}}}{\pi\left(R_{1}^{2}-\mathrm{r}_{\mathrm{m}}^{2}\right)}=2 u_{1} \frac{z}{R_{1}\left[1-\left(\frac{r_{\mathrm{m}}}{R_{1}}\right)^{2}\right]}+\frac{Q_{\mathrm{T}}}{\pi R_{1}^{2}\left[1-\left(\frac{r_{\mathrm{m}}}{R_{1}}\right)^{2}\right]},
$$

Integrating (2) assuming a constant radial circumferential and axial velocity in the range $\left[r_{\mathrm{m}}, R_{1}\right]$ under the condition (3), we obtain $M_{\mathrm{z}}-M_{0}=2 \pi \rho v_{1} u_{1} R_{1}^{2} z$ and

$$
W_{\mathrm{zM}}=3 u_{1} \frac{z}{R_{1}} \frac{1}{1-\left(\frac{r_{\mathrm{m}}}{R_{1}}\right)^{3}}+\frac{3 Q_{\mathrm{r}}}{2 \pi R_{1}^{2}\left[1-\left(\frac{r_{\mathrm{m}}}{R_{1}}\right)^{3}\right]} .
$$

Circulation flow in the axial region is the relation

$$
\mathrm{Q}_{\mathrm{L}}=\left(W_{\mathrm{zM}}-W_{\mathrm{zQ}}\right) \pi R_{1}^{2}\left[1-\left(\frac{r_{\mathrm{m}}}{R_{1}}\right)^{2}\right]
$$

\section{Conclusion}

The peripheral and central zone in a counter-flow cyclone are interconnected through intensive turbulent transfer of angular momentum from the central area to the periphery. Moreover, the generation of turbulence is carried out throughout the chamber.

The proposed ratio allow for evaluation of the flow parameters in the peripheral, pritortsevoy and the axial region of the cyclone chamber.

The work was realized within the research state assignment "Science” №13.1339.2014/K (Code of Federal Target Scientific and Technical Program 2.1410.2014).

\section{References}

1. V.K. Shchukin, A.A. Khalatov, Heat transfer, mass transfer and hydrodynamics of swirled flows in axisymmetrical channels (M .: Engineering 1982)

2. I.I. Smulsky, Aerodynamics and processes in vortexc cameras (Novosibirsk: "Science", 1992)

3. A. Gupta, D. Lillie, N. Sayred Swirling flow (M .: World, 1987)

4. M.A. Goldshtik, A.K. Leontiev, I.I. Belyaev, Heating energy, (2), 40 (1961)

5. S.S. Kutateladze, E.P. Volchkov, V.I. Terekhov, Aerodynamics and heat and mass transfer in limited eddy potokah (Novosibirsk, ITP SB RAS, 1987)

6. S.V. Karpov, E.N. Saburov, TOKCT, 24 (5), 651 (1990)

7. E.N. Saburov, S.V. Karpov, S.I. Ostashev, Heat transfer and aerodynamics of the swirling flow in the cyclone unit (Leningrad: publisher Leningrad University Press, 1989)

8. A.A. Ovchinnikov, N.A. Nikolaev, Proceedings of the Kazan chemical technological institute, 51, 9 (1993)

9. V.A. Borodin, Y.F. Dityakin, L.A. Klyachko, V.I. Yagodkin, Liquid atomization. (Moscow: Mechanical Engineering, 1967)

10. S.V. Alekseenko, P.A. Kuybin, V.L. Okulov, Introduction to the theory of concentrated vortices (Novosibirsk Institute of Thermal Physics SO RAN, 2003)

11. J. Smith, Technical mechanics, 84 (4), 229 (1962)

12. M.I.. Birger, A.Y.Waldberg, B.I. Soft and al., Handbook of dust and ash collection (M .: Energoatomizdat, 1983)

13. B.P. Volodya, Of turbulent transport in rotating flows (Alma-Ata: Science, 1977)

14. V.A. Schwab, Engineering Journal of Physics, 6 (2), 102 (1963)

15. M.V. Wasilewski, Dedusting gas inertial devices (Tomsk: Publishing of Tomsk Polytechnic University, 2008) 